REPLY

\title{
Probiotics, bile acids and gastrointestinal carcinogenesis
}

\section{Wei Jia and Guoxiang Xie}

We thank Borja Sánchez (Bile acid-microbiota crosstalk in gastrointestinal inflammation and carcinogenesis: a role for bifidobacteria and lactobacilli? Nat. Rev. Gastroenterol. Hepatol. https://doi.org/10.1038/nrgastro.2018.23 $(2018))^{1}$ for his interest in our recent Review (Bile acid-microbiota crosstalk in gastrointestinal inflammation and carcinogenesis. Nat. Rev. Gastroenterol. Hepatol. 15, 111-128 $(2018))^{2}$.

In his Correspondence, Sánchez pointed out that probiotic bacteria, bifidobacteria and lactobacilli, could accumulate primary unconjugated bile acids (BAs) such as cholic acid (CA) and probably secondary unconjugated BAs such as deoxycholic acid (DCA) in their cytoplasm either spontaneously or following intracellular BA deconjugation ${ }^{3,4}$. The primary BAs sequestered in the cytoplasm of these bacteria might escape from the portal circulation and the action of other microorganisms to produce secondary BAs. Thus, a BA depletion strategy using probiotics could be a useful means of reducing colonic DCA levels, leading to decreased inflammation and gastrointestinal cancer risk. This concept is certainly an interesting topic for discussion.

However, we believe that it is premature to have included results from an in vitro study ${ }^{5}$ in our Review. First, BAs are reabsorbed primarily in the small intestine (terminal ileum), whereas intestinal BA depletion by bacteria is likely to occur in the large intestine where bacteria such as bifidobacteria and lactobacilli are present in sufficient amounts to demonstrate the effect of lowering intestinal BA concentrations. Probiotics such as Lactobacillus and Bifidobacterium spp. are primarily located in the large intestine, which contains the highest bacterial density, about $10^{11}-10^{12}$ bacteria per gram of intestinal content ${ }^{6,7}$. In other words, BA binding by probiotics that occurs predominantly in the large intestine will probably not affect the apical sodium-dependent BA transportermediated BA reabsorption that takes place in the upper intestine. Thus, the proposed BA removal strategy via probiotics might not be practically achievable in vivo. Secondly, the ability of a given probiotic to bind and release BAs has not been well studied in vivo. Unlike the action of a BA sequestrant resin such as cholestyramine, the probiotic strains must grow to achieve a sufficient amount in the intestine for the binding to become substantial and have notable effects. Although BAs are absorbed by some of the intestinal bacteria, for example, CA is accumulated spontaneously in energized probiotic bacteria $^{3,4}$, BAs in the cytoplasm of intestinal bacteria will be partially released into the intestinal lumen. Lactococcus lactis has been reported to actively release CA via a multispecific organic anion transporter ${ }^{8}$. Thus, well-characterized probiotic strains with a strong ability for BA uptake but not $\mathrm{BA}$ excretion into the gastrointestinal tract could potentially be useful in this application. Additionally, oral administration of BA-binding probiotics with high bile salt hydrolase activity could increase BA deconjugation in the small intestine and, therefore, facilitate greater reabsorption of deconjugated BAs from the distal ileum. Taken together, it is not clear, at this point, how probiotics interact with BAs in vivo and whether they have a beneficial effect in depleting cytotoxic secondary BAs in the gastrointestinal tract. We will have the answers to these questions only after in-depth mechanistic studies have been performed, particularly in vivo.

Wei Jia ${ }^{1,2 *}$ and Guoxiang Xie ${ }^{1,2}$

${ }^{\prime}$ Center for Translational Medicine, Shanghai Jiao Tong University Affiliated Sixth People's Hospital, Shanghai,

China.

${ }^{2}$ University of Hawaii Cancer Center, Honolulu, HI, USA. *e-mail:wjia@cc.hawaii.edu doi: $10.1038 /$ nrgastro.2018.24 Published online 7 Mar 2018

1. Sănchez, B. Bile acid-microbiota crosstalk in gastrointestinal inflammation and carcinogenesis: a role for bifidobacteria and lactobacilli? Nat. Rev. Gastroenterol. Hepatol. https://doi.org/10.1038/ nrgastro.2018.23 (2018).

2. Jia, W., Xie, G. \& Jia, W. Bile acid-microbiota crosstalk in gastrointestinal inflammation and carcinogenesis. Nat. Rev. Gastroenterol. Hepatol. 15, 111-128 (2018).

3. Kurdi, P. et al. Cholic acid is accumulated spontaneously, driven by membrane $\Delta \mathrm{pH}$, in many lactobacilli. J. Bacteriol. 182, 6525-6528 (2000).

4. Kurdi, P. et al. Cholic acid accumulation and its diminution by short-chain fatty acids in bifidobacteria. Microbiology 149, 2031-2037 (2003).

5. Guo, C. F. et al. Screening for cholesterol-lowering probiotic based on deoxycholic acid removal pathway and studying its functional mechanisms in vitro. Anaerobe 18, 516-522 (2012).

6. Ley, R. E., Peterson, D. A. \& Gordon, J. I. Ecological and evolutionary forces shaping microbial diversity in the human intestine. Cell 124, 837-848 (2006).

7. Ridlon, J. M., Kang, D. J. \& Hylemon, P. B. Bile salt biotransformations by human intestinal bacteria. J. Lipid. Res. 47, 241-259 (2006).

8. Yokota, A. et al. Cholate resistance in Lactococcus lactis is mediated by an ATP-dependent multispecific organic anion transporter. J. Bacteriol. 182 5196-5201 (2000).

Competing interests

The authors declare no competing interests. 